Territoire en mouvement Revue de géographie et aménagement

Territory in movement Journal of geography and planning

$13 \mid 2012$

Visibilité et invisibilité, emplois et réemplois du religieux

\title{
La rénovation urbaine comme occasion de réduire les inégalités face à la sécurité des déplacements : une comparaison franco-allemande
}

Urban renewal as opportunity to reduce road safety inequalities: a comparison between France and Germany

Sylvanie Godillon

\section{revues.org}

Édition électronique

URL : http://tem.revues.org/1639

DOI : 10.4000/tem.1639

ISSN : $1950-5698$

\section{Éditeur}

Université des Sciences et Technologies de Lille

Édition imprimée

Date de publication : 1 mai 2012

Pagination : 163-176

ISSN : 1954-4863

\section{Référence électronique}

Sylvanie Godillon, «La rénovation urbaine comme occasion de réduire les inégalités face à la sécurité des déplacements : une comparaison franco-allemande ", Territoire en mouvement Revue de géographie et aménagement [En ligne], 13 | 2012, mis en ligne le 01 janvier 2014, consulté le 25 octobre 2016.

URL : http://tem.revues.org/1639; DOI : 10.4000/tem.1639

Ce document est un fac-similé de l'édition imprimée.

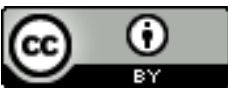

Territoire en mouvement est mis à disposition selon les termes de la licence Creative Commons Attribution 4.0 International. 


\title{
La rénovation urbaine comme occasion de réduire les inégalités face à la sécurité des déplacements :
}

\section{une comparaison franco-allemande}

\author{
Sylvanie GODILLON \\ IFSTTAR - MA \\ 23 rue Nobel \\ 77420 CHAMPS-SUR-MARNE \\ godillon_sylvanie@yahoo.fr
}

\section{Résumé}

Des recherches récentes montrent que les quartiers défavorisés enregistrent davantage d'accidents de la route que les autres. Les facteurs avancés sont la faible part d'espaces de loisirs, d'importants niveaux de trafic et l'absence de traversées sécurisées pour les piétons. Agir sur la morphologie urbaine serait donc un moyen d'améliorer la sécurité. Dans le cadre de programmes nationaux de rénovation urbaine, les projets urbains ont des impacts sur les espaces publics et l'organisation du trafic dans le quartier. Les responsables sont-ils conscients de leur influence en termes de sécurité des déplacements?

L'article s'intéresse à l'articulation de la sécurité routière et des projets urbains menés lors des programmes de rénovation urbaine. Les projets urbains sont-ils en contradiction avec la sécurité routière?

Une comparaison avec l'Allemagne est pertinente pour analyser les différentes manières de coordonner la rénovation urbaine avec la sécurité routière en raison d'une plus grande souplesse de l'échelle locale en Allemagne. Les mécanismes de coordination sont décryptés à deux échelles : les orientations sont fixées par l'échelle nationale et la mise en ouvre est locale.

Deux différences principales sont identifiées. Premièrement, en Allemagne les intérêts de la sécurité routière et du projet urbain convergent avec un trafic qui contourne le quartier, alors qu'en France les intérêts divergent en raison d'une forte volonté de rendre le quartier traversant. Deuxièmement, les spécialistes de la sécurité routière sont présents lors des définitions de projet urbain en Allemagne, alors qu'ils interviennent dans la réalisation du projet en France.

La rénovation urbaine est une occasion d'intégrer des enjeux de sécurité des déplacements lors du réaménagement des quartiers. Si cette occasion est exprimée par les acteurs locaux, elle reste difficile à saisir en France en raison d'une tardive implication des spécialistes de la sécurité routière dans les conduites de projet de rénovation urbaine par rapport à l'Allemagne.

Mots-clés : rénovation urbaine, projet urbain, sécurité routière, France, Allemagne

\begin{abstract}
Recent research shows that road traffic accidents occur more often in deprived neighbourhoods than in other areas. The factors proposed are the small proportion of recreation areas, the high traffic level and the lack of safe crosswalks. Security could thus be improved by redeveloping the urban fabric. As parts of national urban renewal programs, urban projects impact on public spaces and traffic in the neighbourhoods. Are the projects managers aware of the way they influence traffic safety?

This paper focuses on the link between traffic safety and urban renewal projects, and on the issue of a possible contradiction between them.

The flexibility of the local local authorities in Germany makes it useful to compare different ways of coordinating urban renewal with traffic safety. Coordination mechanisms are analyzed at two different scales: the national elaboration of guidelines, and the implementation of local projects.

Two main differences can be identified. First, in Germany both traffic safety policies and urban projects aim at making the traffic bypass the neighbourhood, whereas the will to make the traffic cross neighbourhoods often prevails in France. Second,traffic safety experts in Germany play a part in the determining of urban projects, whereas they intervene at the stage of implementation in France.

Urban renewal is an opportunity to integrate traffic safety issues into neighbourhoods' redevelopment. Local actors are aware of this opportunity and ask for this process, but in France it remains difficult to understand, given that traffic safety experts have gotten involved in the management of urban renewal projects much later than in Germany.
\end{abstract}

Keywords: urban renewal, urban project, traffic safety, France, Germany 


\section{INTRODUCTION}

En 2006, les chercheurs Aguero-Valverde et Jovanis, de l'Institut des Transports de Pennsylvanie (Etats-Unis), montrent de fortes corrélations spatiales entre les accidents, les niveaux socio-économiques, les conditions climatiques, les infrastructures de transport et les niveaux de trafic. Les quartiers, avec une importante part de population vivant en dessous du seuil de pauvreté et avec une forte proportion d'habitants âgés de moins de 25 ans et de plus de 64 ans, enregistrent davantage d'accidents de la route (AGUERO-VALVERDE et JOVANIS, 2006).

Ces résultats font écho à des recherches plus anciennes. La britannique Preston montre, en 1972, que les zones les plus accidentogènes sont souvent associées à une forte concentration de l'habitat avec de petits jardins voire sans jardin, des accès aux bâtiments longeant les voies, aucun espace pour les jeux d'enfants, et d'importants trafics de transit à travers le quartier. Les liens entre les concentrations d'accidents, l'absence d'espaces verts et les trafics importants sont confirmés, en 1991, par les canadiens Joly, Foggin et Pless.

La morphologie du quartier joue donc un rôle important dans l'explication de l'insécurité des déplacements. Or, les quartiers défavorisés sont la cible de programmes de rénovation urbaine ayant des objectifs sociaux et urbains. L'objectif principal est de réduire les inégalités sociospatiales. Aucune mesure n'est affichée pour réduire les inégalités socio-spatiales face au risque routier alors que ces projets sont l'occasion de les traiter. En effet, un des objectifs vise l'amélioration de la qualité du cadre de vie, à travers notamment une requalification des espaces publics. Par ces actions, les pouvoirs publics peuvent améliorer la sécurité routière dans les quartiers défavorisés et réduire les différenciations entre les quartiers face à cette insécurité. Un autre objectif est de réduire l'enclavement des quartiers défavorisés en créant des liens avec les quartiers voisins. Cet objectif d'ouverture n'est-il pas en contradiction avec des objectifs de sécurité routière?

Alors que l'insécurité routière est souvent renvoyée à la responsabilité individuelle, un des moyens d'action pour lutter contre l'insécurité routière est une action sur l'environnement (FLEURY, 1998). Les erreurs de conduites sont analysées ici comme la résultante d'un système formé par l'individu, le mode de transport et l'environnement (VAN ELSLANDE et al., 1997). Concernant l'environnement précisément, Marine Millot démontre que l'organisation des réseaux routiers, la disposition du bâti, les caractéristiques visuelles de l'environnement, les aménagements de l'espace public sont des éléments ayant un impact sur la sécurité des déplacements (MILLOT, 2003). Ainsi, la sécurité routière est portée à la fois par les services techniques, qui traitent explicitement des accidents, et par des politiques urbaines plus globales, qui influencent implicitement la sécurité. L'intégration des services traitant de la sécurité des déplacements en amont du projet permet une meilleure prise en compte des spécificités de déplacements (FLEURY, 1998 ; MILLOT, 2003). L'article s'intéresse à l'articulation de la sécurité routière et des projets urbains menés lors des programmes de rénovation urbaine. Les objectifs des projets urbains sont-ils en contradiction avec ceux de sécurité routière?

Sur la problématique de coordination des politiques de transport et d'urbanisme, une approche comparative avec l'Allemagne est pertinente. La responsabilité des autorités locales facilite davantage de souplesse et de prise en compte des spécificités locales (BLANC, 2006), ce qui laisse supposer une coordination facilitée. Les contextes socio-économiques et politiques sont comparables dans les deux pays. En Allemagne, le programme Soziale Stadt a pour objectif de développer les quartiers défavorisés. L'intérêt d'une comparaison réside également dans la méthode : "le "détour" comparatif permet de porter un regard décentré sur sa propre réalité nationale, en questionnant des éléments qui peuvent paraître évidents d'un point de vue strictement interne " (HASSENTEUFEL, 2005) et " l'exercice de la dénaturalisation et de comparaison oblige à formuler des hypothèses neuves, d'où son intérêt intellectuel » (LIMA, 2004).

La difficulté de la démarche est de saisir l'objet "sécurité des déplacements ". Cet objet s'inscrit à plusieurs échelles puisque les orientations sont fixées par l'échelle nationale mais que sa mise en ouvre est locale. Cette double inscription concerne également la rénovation urbaine. Léchelle nationale et l'échelle locale sont considérées afin d'analyser la prise en compte des enjeux 
de sécurité dans les programmes de rénovation urbaine. Textes de lois, orientations des programmes, description des projets urbains, et analyse des discours des acteurs sont les matériaux utilisés pour saisir la sécurité routière à différentes échelles. En outre, la sécurité des déplacements se trouve dans plusieurs champs : la sécurité routière analyse les accidents (sécurité explicite) et les politiques urbaines traitent des espaces publics, des déplacements ou du partage de la voirie dans un souci d'amélioration de la qualité de vie en ville (sécurité implicite).

Les différentes manières de prendre en compte les enjeux de sécurité routière dans les projets urbains en direction des quartiers défavorisés sont ainsi croisées à plusieurs échelles et thématiques. Après avoir confronté les deux programmes nationaux de rénovation urbaine et les politiques de sécurité routière, il s'agira de confronter la mise en ouvre de ces deux programmes à l'échelle locale, celle des quartiers de projets dans l'agglomération de Lille en France et à Dresde en Allemagne.

\section{PORTRAit de DEUX POLITIQUeS NATIONALES}

\subsection{La rénovation urbaine en Allemagne et en France}

Le terme de "rénovation urbaine ", utilisé dans cet article, fait référence aux politiques à la fois sociales et urbaines en direction des quartiers défavorisés comme le Programme National de Rénovation Urbaine en France depuis 2003 et le programme Soziale Stadt en Allemagne depuis 1999.

L'avènement d'une politique de rénovation des quartiers défavorisés

Dans les deux pays, les années 1970 marquent les prémisses d'une politique urbaine et sociale en direction des espaces urbains défavorisés. Dans l'ancienne République Fédérale Allemande (RFA), les années 1970 ont vu des politiques de réhabilitation urbaine (Sanierung) se mettre en place. Par contre, il faut attendre la réunification de l'Allemagne pour la mise en place de telles politiques dans l'ancienne République Démocratique Allemande (RDA). En France, dès le milieu des années 1970, le programme « Habitat et vie sociale » est lancé par l'Etat et des bailleurs de logements sociaux pour améliorer l'habitat dans les quartiers construits rapidement après la Seconde Guerre mondiale. Ce programme comporte également un volet social. Le changement de couleur politique nationale et des incidents de violence urbaine incitent une politique plus volontariste au début des années 1980. Le rapport Dubedout (1982), Ensemble, refaire la ville, est à l'origine de la politique de Développement Social des Quartiers (DSQ) dont l'action est fondée sur quatre axes : agir sur la dégradation des quartiers et les causes de cette dégradation, faire des habitants les acteurs du changement, rendre la collectivité locale responsable des opérations, faire assumer à l'Etat son devoir de solidarité nationale. La planification est contractuelle : l'Etat et les collectivités choisissent les sites prioritaires.

A partir des années 1990, le volontarisme étatique s'affirme. En France, cette tendance est marquée par la nomination d'un ministre en charge de la politique de la ville en 1990 et par la mise en place du pacte de relance pour la ville en 1996. Au début des années 2000, la loi d'orientation sur la ville et la rénovation urbaine donne le ton de l'actuelle politique en direction des quartiers défavorisés, avec des mesures alliant le social et l'urbain. En Allemagne, au lendemain de la réunification, dès les années 1990, quelques Länder ${ }^{1}$ (notamment la Rhénanie du Nord-Westphalie et Hambourg) développent des politiques urbaines sociales et expriment le souhait d'une prise de position fédérale concernant les quartiers défavorisés. La conférence des ministres des Länder chargés de l'urbanisme, de la construction et de l'habitat (ARGEBAU) a élaboré l'initiative. En 1999, le programme Stadtteile mit besonderem Entwicklungsbedarf - Soziale Stadt ("Ville sociale, quartiers avec un besoin particulier de développement ») est lancé. En Europe, les idées circulent. Maurice Blanc, professeur à l'Institut d'urbanisme de Strasbourg et chercheur associé au centre Marc Bloch de Berlin, montre que le programme allemand s'appuie sur des principes développés par le Royaume-Uni pour la régénération économique et la doctrine sociale française, même si la France ne la met pas réellement en pratique (BLANC, 2006).

${ }^{1}$ En Allemagne, les Länder sont des Etats, fédérés dans un Etat Fédéral : le Bund. 
Ainsi, en France et dans l'ouest de l'Allemagne, différentes politiques pour améliorer les conditions de vie des habitants des quartiers défavorisés sont menées depuis les années 1970. Ces politiques ont comme point commun de proposer des mesures à la fois sociales et urbaines pour développer les quartiers en difficultés (BLANC, 2006). Les mesures urbaines impactent l'organisation du bâti et de la trame viaire, et donc l'aménagement des déplacements.

Un objectif de la politique actuelle concerne les espaces publics

Les objectifs des deux programmes nationaux sont de réduire les écarts en aidant le développement des quartiers les plus défavorisés. En France, l'objectif principal est la réduction des inégalités entre les territoires et la lutte contre l'exclusion. La loi d'orientation et de programmation pour la ville et la rénovation urbaine ( $\mathrm{du}$ $1^{\mathrm{er}}$ août 2003) a pour objectif de réduire les écarts entre les quartiers considérés sous l'angle de leurs handicaps urbains (morphologie, niveau d'équipement, enclavement) et sociaux (concentration de populations défavorisées). L'ambition pour le Programme National de Rénovation Urbaine est de transformer les quartiers les plus en difficultés et d'améliorer les conditions de vie des habitants. En Allemagne, l'objectif principal est une mobilisation de l'ensemble des acteurs (publics et privés) pour le développement du quartier. En 2004, le code de la construction (Baugesetzbuch) est modifié et intègre les éléments de la loi de rénovation urbaine, ainsi que les dispositions relatives au programme Soziale Stadt ${ }^{2}$. Les mesures prises dans le cadre du programme Soziale Stadt s'appuient donc sur des mesures urbaines et pas directement sur des mesures sociales.

Les programmes sont proches avec une place importante accordée à l'urbanisme. En France, la démarche se fonde sur une rénovation de l'habitat et des espaces publics, une dynamisation des commerces de proximité et des actions en faveur du développement économique et de l'emploi. Le sociologue Renaud Epstein démontre que les outils mis en place sont entièrement tournés vers cet objectif : la restructuration urbaine des quartiers et la réduction de la concentration spatiale des populations défavorisées sont facilitées par les démolitions (EPSTEIN, 2005).

\footnotetext{
${ }^{2}$ Paragraphe 171 du code de la construction.
}

La transformation des quartiers passe par une transformation des espaces publics de déplacements et ces actions ont donc un impact sur les possibilités d'amélioration de la sécurité des déplacements dans le quartier. En Allemagne, le champ politique de l'urbanisme a une position de leader et les autres ministères fédéraux doivent concentrer leurs nouveaux programmes sur les quartiers concernés par le programme Soziale Stadt (JACQUIER, 2003). Les champs d' action sont 1 'emploi, 1 ' habitat, les activités culturelles, l'école, les espaces publics et de proximité, la santé, l'environnement et les transports. Ainsi, le développement des quartiers passe par des mesures urbaines, dont une mesure précise concerne les espaces publics.

Les programmes de rénovation urbaine français et allemand se ressemblent au sens où ils proposent des mesures sociales et urbaines et où l'urbanisme fait figure de leader. Ces programmes financent des projets urbains, dont une part est dédiée aux espaces publics. Or ces espaces publics sont le lieu des déplacements dans le quartier : les problématiques de déplacements sont donc en filigrane de ces programmes.

Coordonner la rénovation urbaine avec les politiques de déplacements

Dans les deux pays, les politiques sectorielles (emploi, culture, éducation, transport...) menées dans le cadre de ces politiques urbaines doivent être coordonnées. En France, les principes de cohérence entre les documents d'urbanisme et les exigences de concertation entre les acteurs permettent théoriquement des ponts entre le Programme National de Rénovation Urbaine et les politiques de transports. Les actions relatives à la politique de la ville tiennent compte des autres documents de planification à l'œuvre sur le territoire, notamment le Plan de Déplacements Urbains qui a des objectifs en termes de sécurité des déplacements depuis la circulaire Meltt de 1997 (DESIRE et MONTEL, 2000), réaffirmés par la loi Solidarité et Renouvellements Urbains du 13 décembre 2000. En Allemagne, la notion d'intégration entre les actions est présente dans les différentes politiques sectorielles : au niveau général de la planification urbaine, le schéma de développement urbain intégré (Integrierte Stadtentwicklungskonzept) a pour objectif de croiser 
les différentes politiques ; dans le domaine des transports, le schéma intégré des transports (Integrierte Verkehrskonzept) vise à tenir compte du développement urbain et des enjeux de pollution, de bruit et d'insécurité routière. Ces documents ne sont pas une obligation légale mais incitent les coordinations entre les différents politiques sectorielles (AHRENS, 2008).

Le programme Soziale Stadt impose des outils pour coordonner les politiques sectorielles, contrairement au Programme National de Rénovation Urbaine. Les principes de la loi du $1^{\text {er }}$ août 2003 sur la rénovation urbaine sont d'impliquer au plus tôt les habitants dans une logique de concertation et de renforcer les partenariats entre les acteurs locaux concernés par le projet urbain (bailleurs, Etat, collectivités, ...). Pour cela, une convention est passée entre l'Agence Nationale de la Rénovation Urbaine (ANRU) et les différents acteurs. Dans les textes, la coordination entre les projets de rénovation urbaine et les objectifs de transport est donc assurée. Le programme Soziale Stadt va plus loin. En effet, la loi relative au programme Soziale Stadt définit les fondements d'un développement coordonné entre les différents acteurs. La loi impose une mobilisation de tous les acteurs pour le développement du quartier et propose deux outils concrets : le projet d'action intégré (Integriertes Handlungskonzept) et le management de quartier (Quartiermanagement). Le projet d'action intégré permet de définir et de coordonner les différents champs d'action concernés au moment de la définition et de la mise en œuvre du projet. Concrètement, ce projet a la forme $d$ 'un document d'une centaine de pages diagnostiquant les problèmes et proposant des réponses. Ce projet d' action intégré permet une approche globale. L'autre outil permettant une approche globale est le management de quartier (Quartiermanagement), chargé d'assurer le lien entre les différents acteurs et d'impliquer le public. Concrètement, le management de quartier prend forme dans 1'organisation de réunions et de tables rondes. La mobilisation des ressources doit permettre de combiner et d'accorder les projets des quartiers concernés (BECKER et al., 2003).
Dans les textes, les deux programmes de rénovation urbaine prônent la coordination entre les acteurs agissant sur le même espace défavorisé pour améliorer les conditions de vie des habitants. Dans les deux pays, les programmes de rénovation urbaine doivent faire référence aux autres documents de planification, notamment ceux relatifs aux déplacements et à la sécurité. Théoriquement, les objectifs de sécurité routière sont donc repris dans les projets d'aménagements. Reste à voir si les objectifs de sécurité routière sont spatialisés.

\subsection{La sécurité des déplacements en Allemagne et en France}

De grandes orientations tournées vers les responsabilités individuelles

Dans son livre blanc « La politique européenne des transports à l'horizon 2010 : l'heure des choix " (COMMISSION EUROPEENNE, 2001), l'Union Européenne fixe comme objectif la réduction de moitié du nombre de tués. Deux actions sont entreprises à l'échelle de l'Union Européenne : l'harmonisation des sanctions et la promotion des nouvelles technologies au service de la sécurité routière. Le choix de ces deux actions, notamment l'importance accordée aux sanctions, montre que la sécurité routière est fortement renvoyée à la responsabilité individuelle.

Dans les deux pays étudiés, ce mot d'ordre se retrouve. En France, le comité interministériel de la sécurité routière (CISR) a pour mission de définir la politique du gouvernement en matière de sécurité routière et de s'assurer de son application. La mise en ouvre de la politique locale de sécurité routière par l'État est placée sous la responsabilité du préfet de chaque département. Ce comité rassemble différents ministères concernés par la sécurité routière : Intérieur, Défense, Éducation nationale, Justice, Santé et Travail ${ }^{3}$. Les différents ministères présents dans ce comité montrent l'importance accordée aux responsabilités individuelles puisque le ministère en charge du transport n'est pas représenté. En Allemagne, les grandes orientations sont données au niveau fédéral. Le programme fédéral, en 2010, poursuit cinq priorités : améliorer les conditions de circulation, protéger les

\footnotetext{
${ }^{3}$ Site du Ministère de l'Ecologie, de l'Energie, du Développement Durable et de la Mer -

http://www.securiteroutiere.gouv.fr/ - consulté le 25 novembre 2011
} 
usagers vulnérables, réduire le risque d'accidents chez les jeunes, réduire le danger des véhicules lourds et améliorer la sécurité en milieu rural. Le programme de sécurité routière est élaboré au niveau du Land ${ }^{4}$ qui définit les lignes directrices d'un programme de sécurité routière.

En Allemagne comme en France, les grandes orientations insistent sur les responsabilités individuelles.

Coordonner la sécurité des déplacements avec la rénovation urbaine?

En France et en Allemagne, les politiques de sécurité routière nationales ont des objectifs généraux renvoyant davantage aux comportements individuels qu'à des politiques d'aménagements des infrastructures.

Les liens avec l'urbanisme des quartiers traversés par les réseaux ne sont pas tissés. Dans les textes de lois, les circulaires, les programmes concernant la sécurité routière, très peu de références sont faites aux programmes de rénovation urbaine. En France, deux études du Centre d'Etudes sur les Réseaux, le Transport et l'Urbanisme (CERTU) traitent de l'insécurité des déplacements dans les quartiers défavorisés. La première, réalisée avec la Délégation Interministérielle à la Ville (DIV) en 1996, pose l'insécurité comme un enjeu majeur du renouvellement urbain dans les quartiers défavorisés et propose des recommandations telles que la hiérarchisation et l'affectation des espaces en fonction de leur usage. La seconde, réalisée en 2008 avec le CETE Méditerranée, identifie les spécificités de l'insécurité routière dans quatre quartiers de la politique de la ville. En Allemagne, un rapport de l'Institut allemand d'urbanisme (Deutsches Institut für Urbanistik - DIFU) sur les stratégies du programme Soziale Stadt aborde la question des accidents comme une des composantes des inégalités écologiques et se réfère aux travaux d'un spécialiste allemand de la justice environnementale : Werner Maschewsky (DIFU, 2003). Cette littérature reste très limitée en nombre.

La rénovation urbaine est impulsée par l'Etat dans les deux pays mais prend forme à l'échelle des quartiers accueillant les projets. Quand des améliorations de réseaux sont effectuées, la sécurité des déplacements se matérialise loca- lement, mais il existe peu de documents de référence sur les liens entre l'insécurité et les quartiers défavorisés. Les deux programmes sont mis en ouvre à l'échelle locale et conduits par des acteurs locaux relativement autonomes vis-à-vis de l'échelle nationale.

\subsection{Des acteurs locaux plus autonomes en Alle- magne qu'en France}

L'autonomie des acteurs locaux en matière d'urbanisme

Les acteurs locaux sont plus autonomes en Allemagne qu'en France, et ce depuis plus longtemps. En France, les lois de décentralisation depuis les années 1980 ont amorcé une plus grande indépendance des acteurs locaux en matière d'urbanisme. Les Plans Locaux d'Urbanisme sont constitués par les communes suivant le principe de compatibilité avec les documents de planification d'échelle supérieure (Département, Région) et avec les autres documents relatifs au territoire comme les Plans de Déplacements Urbains. L'Etat a la capacité d'intervenir dans la définition des règles locales d'urbanisme à travers trois dispositifs décrits par le Code de l'urbanisme : la déclaration de projet, le projet d'intérêt général et l'opération d'intérêt national. Ces procédures montrent les limites de l'autonomie des acteurs locaux en matière d'urbanisme. Dans le système fédéral allemand, l'aménagement est divisé entre le développement urbain (Städtebau) centré sur les tâches opérationnelles au niveau municipal et la planification régionale (Regionalplanung) fondée sur des grands principes directeurs imposés par les Länder et par l'Etat. La constitution garantit aux municipalités le droit d'assumer la responsabilité de l'organisation de leurs affaires locales dans le respect des lois fédérales et des lois des Länder. De fait, la coordination verticale entre le gouvernement fédéral, les Länder, et les municipalités est indispensable à la réussite de toute politique urbaine. Elle permet de traiter les questions de façons descendante (du Bund vers les collectivités locales) et ascendante (des municipalités et des Länder vers le gouvernement fédéral).

L'Etat est très présent en France dans la mise en œuvre des projets de rénovation urbaine. En effet, les acteurs locaux ont peu de marge

${ }^{4}$ Site du Deutscher Verkehrssicherheitsrat e.V. (DVR) - http://www.dvr.de/ - consulté le 25 novembre 2011 
puisque « le soutien de l'ANRU aux projets locaux est ainsi conditionné par la démolition de logements sociaux, solution "tout terrain" pour changer l'image des quartiers " (EPSTEIN, 2005). Malgré les lois de décentralisation, la France reste donc un pays assez centralisé, alors que Maurice Blanc affirme qu'en Allemagne, «la responsabilisation des autorités locales facilite la souplesse et la prise en compte de spécificités locales » dans le cadre du programme Soziale Stadt. Toutefois, l'auteur nuance son propos en citant des évaluations récentes montrant des cloisonnements entre les services avec des difficultés de compréhension entre les différentes cultures professionnelles (BLANC, 2006). Les acteurs locaux en Allemagne sont plus indépendants qu'en France pour mettre en œuvre localement les programmes de rénovation urbaine.

Les mises en ouvre locales des politiques de sécurité

Dans les deux pays, les politiques de sécurité routière se mettent en ouvre localement. En France, le Conseil Général est un acteur important pour la gestion de la sécurité routière. Toutefois, les actions s'effectuent sous l'égide du préfet, représentant de l'Etat. Un plan d'action pour améliorer la sécurité routière est élaboré chaque année à son initiative. Les regroupements communaux et les villes jouent également un rôle important depuis la décentralisation et la loi Solidarité et Renouvellement Urbains conférant aux Plans de Déplacements Urbains des objectifs de sécurité routière. En Allemagne, les mises en œuvre du programme de sécurité routière sont du ressort des Länder et des villes. Ce sont les autorités locales qui mettent en œuvre les principes directeurs de mobilité et de sécurité, et elles sont également compétentes dans le domaine du contrôle de la sécurité, le stationnement et la circulation. «En Allemagne, le contrôle de la police n'est pas organisé par une autorité fédérale centrale mais par les autorités régionales (Länder). La possibilité de transfert du droit de contrôle aux autorités locales dépend donc du cadre légal particulier à chaque Land (...). Les autorités locales allemandes sont donc en position de contrôler non seulement le stationnement mais aussi la circulation" (KOSSMANN, 2002).
Dans les deux pays, les mises en ouvre du programme de sécurité se font à deux échelles : celles des Länder/départements ${ }^{5}$ et celles des villes. Le préfet est très présent dans les mises en œuvre locales de cette politique en France alors que les Länder sont autonomes en Allemagne. La différence majeure entre les deux pays est que le fédéralisme allemand permet une plus large autonomie des acteurs du Land par rapport au système déconcentré français sous l'autorité du préfet. Le Programme National de Rénovation Urbaine en France et le programme Soziale Stadt en Allemagne sont deux programmes ayant des objectifs sociaux et urbains, notamment concernant les espaces publics. A l'échelle nationale, les objectifs de ces deux politiques ne sont pas contradictoires, l'une cherchant à améliorer le cadre de vie et la qualité des espaces publics dans les quartiers défavorisés et l'autre à réduire les risques d'accidents.

Après avoir comparé les programmes de rénovation urbaine et les politiques de sécurité routière en France et en Allemagne, deux exemples de rénovation urbaine sont confrontés afin de comprendre la coordination de ces deux politiques sectorielles à l'échelle locale.

\section{LA RENCONTRE DE DEUX POLITIQUES TERRITORIALISÉES}

Il est indispensable d'étudier des cas concrets de rénovation urbaine afin de comprendre la coordination des projets de rénovation urbaine et les enjeux de sécurité routière. L'analyse de la mise en ouvre locale des objectifs nationaux permet d'appréhender la manière dont les acteurs locaux s'approprient et mettent en pratique les programmes, les différents liens entre les acteurs et les réalisations urbaines concrètes pour améliorer la sécurité des déplacements dans les quartiers défavorisés.

Dans chaque pays, deux localités ont été choisies : Lille Métropole Communauté Urbaine en France et Dresde en Allemagne. La ville de Dresde est deux fois moins peuplée que l'agglomération lilloise (512 000 habitants pour la première contre plus d'un million d'habitants pour la seconde) et également deux fois moins étendue, si bien que

\footnotetext{
${ }^{5}$ Nous mettons ici en parallèle ces deux échelles qui n'ont rien à voir en termes de compétences : les Länder allemands sont de véritables Etats, contrairement aux départements français. Ce rapprochement se justifie pour les mises en ouvre des politiques de sécurité routière comparables à cette échelle dans les deux pays.
} 
les densités de population sont proches (autour de 1600 habitants par $\mathrm{km}^{2}$ ). Dans leur pays respectif, Lille Métropole Communauté Urbaine et Dresde font figures de localités plutôt défavorisées avec des taux de chômage locaux supérieurs aux moyennes nationales : 13,9\% dans l'agglomération lilloise contre 11,5\% en France, 16,1\% à Dresde contre $11 \%$ en Allemagne (Eurostat, pour la période 2003-2006).

Dans ces deux localités, un exemple de projet de rénovation urbaine a été analysé. Ces projets concernent des quartiers défavorisés. Pour cet article, le choix est orienté par l'importance des actions concernant les espaces publics de dépla- cements au sein du quartier. Dans l'agglomération de Lille, le projet ANRU du quartier des Trois Ponts à Roubaix a été étudié car il s'associe au réaménagement d'un boulevard traversant le quartier. A Dresde, le projet étudié dans le quartier de Gorbitz prévoie un réaménagement de boulevard en zone de trafic calme.

D'une part, ces résultats s'appuient sur une analyse des projets urbains sous l'angle des déplacements et de leur sécurité. Les documents qui détaillent les projets urbains (la convention financière pour le cas de Roubaix et le projet d'action intégrée, Integriertes Handlungskonzept, pour le cas de Gorbitz) ont été analysés à l'aide d'une grille

Figure 1 : Situation des quartiers étudiés

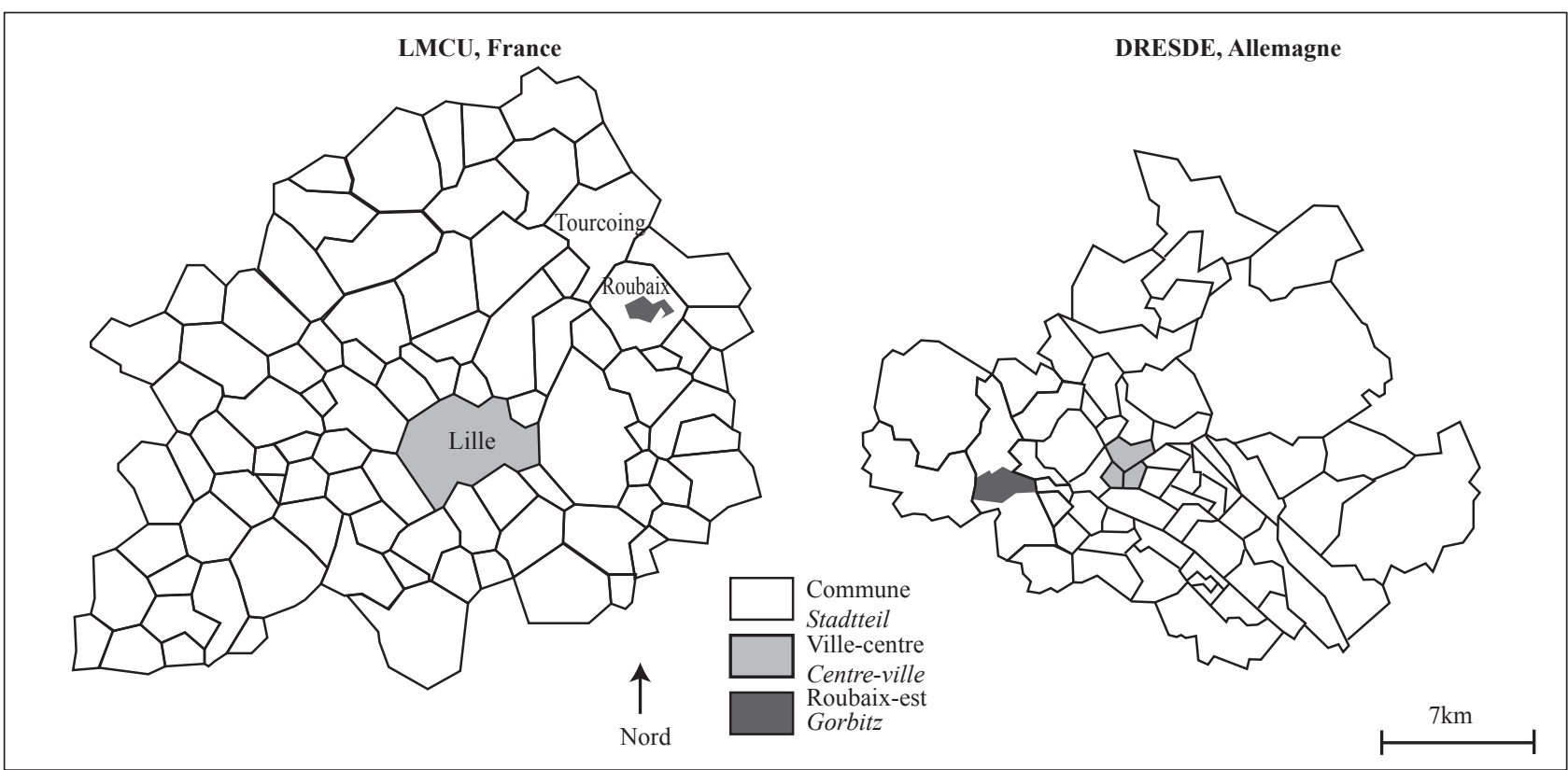

(c) Godillon, 2010.

de lecture à plusieurs entrées : le thème de la sécurité routière est-il abordé ? Si oui, pour quelle cible (enfants, personnes âgées, ...) et quels sont les objectifs? Les projets concernant les voiries ont-ils comme objectif d'améliorer la sécurité ? Comment la question des déplacements est-elle appréhendée? Comment la question du trafic et de l'organisation viaire est-elle traitée?

D'autre part, des entretiens auprès des techniciens des services de mobilité et de sécurité routière, ainsi qu'auprès des responsables des projets urbains de Gorbitz et de Roubaix ont été réalisés ${ }^{6}$, afin de comprendre les représentations et les pratiques de travail des acteurs locaux.

\subsection{Une superposition de projets}

L'amélioration de certains réseaux par la sécurité routière

Les politiques de sécurité se matérialisent localement par des actions pour améliorer la sécurité sur certains réseaux. Toutefois, les objectifs de Dresde ou de Lille Métropole Communauté Urbaine en matière de sécurité routière ne sont pas ciblés sur des quartiers précis comme le précise le directeur du service "Cadre de vie » d'une commune de Lille Métropole Communauté Urbaine : "A l'échelle de la ville, la sécurité est une préoccupation de longue date, à travers des actions sur

${ }^{6}$ Au total, neuf personnes ont été rencontrées : deux au service Voirie et Déplacements à Lille Métropole Communauté Urbaine, deux au service Cadre de Vie de la commune de Roubaix, trois à la planification du développement des transports (Verkehrsenwickungsplanung) à Dresde, la responsable du projet de rénovation urbaine des Trois Ponts à Roubaix et la responsable du projet financé par le programme Soziale Stadt à Gorbitz. 
le comportement et les voiries. Dans les projets ANRU, il n'y a rien de spécifique, la sécurité est un souci partout, il n'y a aucune spécificité ». Les projets pour améliorer la sécurité par des actions sur les infrastructures sont définis sur des réseaux concentrant beaucoup d'accidents.

L'essence des politiques de rénovation est leur inscription spatiale. En effet, depuis les débuts de la politique de la ville en France et dans le programme Soziale Stadt en Allemagne, les projets urbains se définissent à l'échelle d'un quartier dont le périmètre est déterminé par les pouvoirs publics. Ainsi, les deux types de projets se superposent quand les axes très accidentogènes sont dans les quartiers de rénovation urbaine.

La planification du trafic et le réaménagement des rues par la rénovation urbaine

Les projets de rénovation urbaine traitent de la place du trafic dans le quartier en préconisant des voies de contournement (à Gorbitz) ou en diminuant les vitesses de circulation (à Roubaix Est). De plus, les projets de rénovation urbaine réaménagent certaines rues pour améliorer la qualité de vie. Si bien que, même si la sécurité des déplacements n'est pas un objectif explicitement affiché, ces orientations ont des répercussions sur la sécurité des déplacements. Les projets se superposent. Les objectifs de ces deux politiques sont-ils convergents?

\subsection{Une rencontre d'objectifs?}

Améliorer les situations d'insécurité routière

Du côté des responsables des projets urbains, l'objectif est d'améliorer la qualité des déplacements des habitants dans le quartier. En France, la cible est d'emblée les enfants : « auprès des écoles, nous avons de nombreux problèmes ", en précisant que «la sécurité routière est surtout une demande des parents ». Les objectifs sont de répondre aux différentes demandes sociales des habitants, dont la sécurité près des écoles ou les déplacements sécuritaires. A Dresde, les questions de sécurité routière sont impulsées par le constat d'un vieillissement démographique : les personnes âgées ont des besoins spécifiques, notamment concernant la facilité et la sécurité des cheminements au sein de 1'espace résidentiel. Ces représentations s'expliquent par des structures démographiques différentes dans les deux pays avec un vieillissement de la population marqué en Allemagne.
Du côté des services techniques, les projets de rénovation sont une occasion d'améliorer la sécurité par l'obtention de financement. Dans l'agglomération de Lille, les subventions de l'ANRU sont perçues comme un outil financier important : "l'ANRU donne des moyens, l'ANRU est ambitieuse et donne des opportunités pour mener des projets ». Les responsables ne mentionnent pas de travail partenarial formel avec les responsables des projets urbains, contrairement à Dresde où les techniciens affirment qu'ils travaillent régulièrement avec les responsables des projets de rénovation urbaine de manière formelle (réunions, tables rondes) et informelle (demande de conseils sur un quartier en dehors de cadre de réunions). Cette collaboration permet d'avoir des fonds pour financer le réaménagement de certaines rues.

Les objectifs des deux politiques sectorielles convergent : améliorer la sécurité des déplacements des habitants du côté de la rénovation urbaine et améliorer la sécurité sur les réseaux du quartier du côté de la sécurité routière.

Le problème de la place du trafic dans le quartier La convention financière du projet à Roubaix rappelle qu'une des finalités du Plan de Déplacements Urbains (PDU) est de « réduire les nuisances de l'automobile : pollution atmosphérique, bruit, insécurité routière, dégradation de l'espace public » (p. 20). Le projet de rénovation urbaine est l'occasion de mettre en ouvre les objectifs du PDU en permettant « le désenclavement des quartiers " et en réorganisant «le maillage viaire : traversées de ville, liaisons inter quartiers, voies de desserte " (p. 20). Concrètement, le projet dessine de nouvelles lignes de bus à haut niveau de service, un projet de tramtrain, un raccordement des quartiers à la route départementale vers la Belgique, "une stratégie de requalification et de bouclage des boulevards urbains " (p. 21), et une politique de liaisons douces et de promenades urbaines. Les deux dernières propositions peuvent être censées améliorer la sécurité routière, mais le document ne le précise pas et aucune analyse de l'accidentologie n'est proposée. Parallèlement, le projet prévoit d'améliorer l'accessibilité du quartier en créant un « ensemble de pénétrantes automobiles et piétonnes qui incitent à y accéder ou à le traverser " (p. 26). La figure 2 propose une spatialisation de 
ces opérations. Ces créations de voies ne s'accompagnent pas d'une réflexion sur la sécurité routière alors qu'elles ont un impact sur les déplacements et le trafic traversant le quartier. Dans le quartier de Gorbitz à Dresde, le projet d'action intégré (Integriertes Handlungskonzept) indique que grâce aux voies de contournement le trafic dans le quartier est plutôt calme (p. 43). A Gorbitz, deux mesures devraient améliorer la sécurité routière : la mise en place de zones de trafic apaisé (Verkehrsberuhigung ${ }^{7}$ ) et l'amélioration des conditions de traversées piétonnes des boulevards ceinturant le quartier (pages 57-58). Le projet propose d'apaiser le trafic en vue d'améliorer la sécurité routière et ne remet pas en cause la logique originale de contournement du quartier (figure 2). Un moindre trafic dans le quartier est en accord avec moins d'accidents dans le quartier. Dans les deux cas, les solutions proposées sont semblables avec une volonté d'apaiser le trafic et d'améliorer les espaces publics de déplacements, mais aucune analyse de l'insécurité n'est effectuée. En France, les objectifs de désenclavement sont en contradiction avec les objectifs de sécurité routière, alors qu'en Allemagne les deux politiques poursuivent les mêmes objectifs. Quand et comment les acteurs peuvent-ils exprimer leurs objectifs?

Figure 2 : Projet de rénovation urbaine

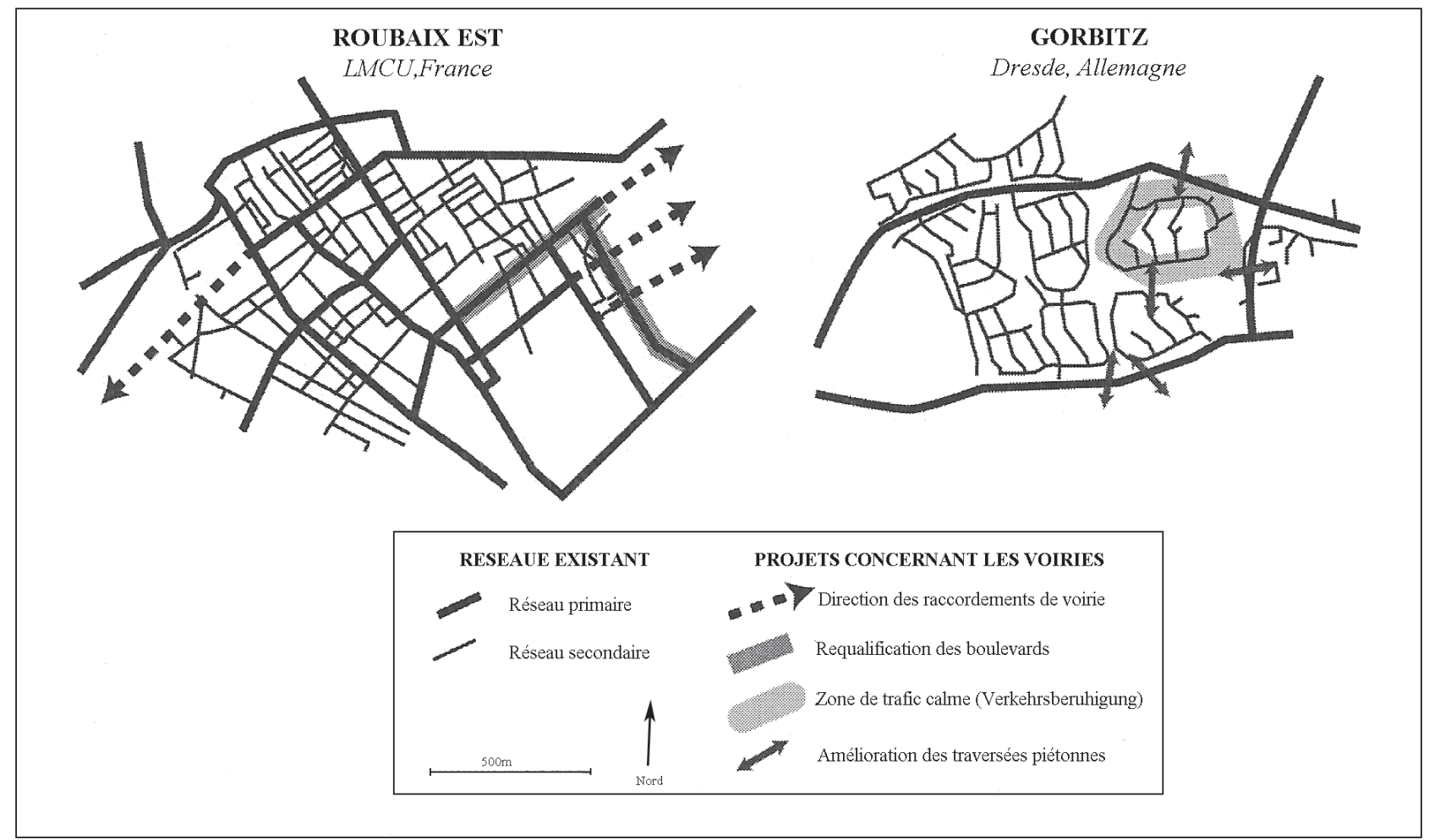

(c) Godillon, 2010.

\subsection{Un partenariat entre les acteurs?}

Une proximité plus étroite entre les différents acteurs à Dresde qu'à Lille

En Allemagne comme en France, les textes de loi des programmes de rénovation urbaine ont pour principe un partenariat étroit entre les acteurs agissant sur les quartiers concernés. Toutefois, les textes ne stipulent pas explicitement l'implication des acteurs de la sécurité routière dans les projets de rénovation urbaine. Les organisations administratives de l'agglomération lilloise et de la commune de Dresde sont ici analysées afin d'étudier la proximité entre les services. La compréhension des relations de travail entre les services sera effectuée dans un second temps.

${ }^{7}$ Le concept de zone de trafic apaisé (Verkehrsberuhigung) est défini par le StVO, le code de la route allemand. Dans une zone de trafic apaisé les piétons peuvent utiliser la voie dans toute sa largeur et les jeux d'enfants sont acceptés partout. Les véhicules doivent se conformer à la vitesse de marche et ne doivent pas mettre en danger ou gêner les piétons, le cas échéant les conducteurs attendent. Cependant, le piéton ne doit pas inutilement entraver la circulation. Les objectifs de ce type de zone sont d'améliorer la sécurité ainsi que le cadre de vie en transformant la rue. 
Trois services sont ciblés : le service s'occupant des projets urbains en direction des quartiers défavorisés, le service organisant la mobilité à l'échelle de l'agglomération lilloise et de la ville de Dresde et les services chargés de mettre en ouvre concrètement les dispositifs visant à réduire le nombre d'accidents.

L'organisation interne de l'administration allemande permet plus de liens entre les acteurs des transports et les acteurs de la rénovation urbaine. A Dresde, le service du développement urbain (Stadtentwicklung) est composé de quatre entités : urbanisme (Stadtplanungsamt), géomètre (Städtisches Vermessungsamt), construction (Bauaufsichtsamt) et les services techniques (Straßen- und Tiefbauamt). L'urbanisme intègre la planification du développement urbain (Stadtentwicklungsplanung) et la planification du développement des transports (Verkehrsentwicklungsplanung). La mise en parallèle avec l'agglomération de Lille permet de constater que le développement urbain (équivalent français du Stadtentwicklung) et les services techniques (équivalent français du Straßen- und Tiefbauamt) sont dans deux services bien distincts : les services techniques et le service Aménagement et cadre de vie (figure 3).

A ceci s'ajoute la complexité d'un système français double : à Lille, la sécurité routière est de compétence métropolitaine alors que la mise en œuvre de la politique de la ville s'effectue à l'échelle de la commune. Cette complexité n'est pas prise en compte dans la figure 3 et est analysée dans la conduite de projet. Sans tenir compte de ce système de double compétence, la proximité des acteurs est plus importante en Allemagne.

Toutefois, cette proximité ne signifie pas forcément que les différents services travaillent ensemble. En effet, "l'existence d'institutions intégrant les compétences de transport et d'urbanisme ne suffit pas à la mise en oeuvre de politiques coordonnées" (GALLEZ et al., 2008 : 128). Il s'agit de vérifier que cette proximité entre acteurs s'accompagne d'une proximité effective lors de la conduite de projet, du diagnostic à la réalisation du projet de rénovation urbaine.

Des spécialistes de la sécurité routière présents en Allemagne

La conduite de projet est organisée en partie par les textes de loi, mais dépend également de l'organisation institutionnelle et des habitudes de partenariat entre les services.
En France, l'agglomération est chargée des questions de sécurité routière alors que la commune assure la mise en œuvre du Programme National de Rénovation Urbaine. De plus, Lille Métropole Communauté Urbaine (LMCU) est le maître d'ouvrage pour la voirie et les transports collectifs et la ville de Roubaix pour les espaces verts et le stationnement. Compte tenu de l'imbrication des compétences entre la ville et Lille Métropole Communauté Urbaine, une convention de groupement de commande entre la Communauté Urbaine de Lille, la ville de Roubaix et la Société d'Economie Mixte Ville Renouvelée a été signée pour une période de cinq ans afin d'optimiser la gestion des interfaces et les relations avec l'ensemble des intervenants. Lors de la définition des projets d'aménagements des espaces publics du quartier des Trois Ponts à Roubaix, le service Voirie et espaces publics de Lille Métropole Communauté Urbaine, au sein duquel sont menées les études d'accidentologie et qui connaît les enjeux, n'est pas présent : "De toute façon, les gens de l'ANRU pensent que c'est du comportement. Regarder les accidents qui se passent dans le quartier est un réflexe qu'ils n'ont pas et qu'ils pourraient avoir ». Les services techniques de la ville ne sont également pas présents et interviennent après en leur qualité de maître d'ouvrage, une fois le projet urbain défini (figure 4).

En Allemagne, la sécurité routière et le programme Soziale Stadt sont conduits par la ville de Dresde, mettant en lumière cette spécificité française de multiplicité de couches de décisions au niveau local. L'obligation du programme Soziale Stadt de réunir l'ensemble des acteurs afin de mobiliser les ressources d'un territoire incite à la présence des services techniques lors de la définition des projets d'aménagements des espaces publics (figure 4). Ainsi, en mai 2008, une table ronde sur les espaces publics a réuni à la fois les responsables du projet urbain et les personnes en charge de la sécurité routière. Dans le cadre de l'identification des objectifs et de la définition du projet urbain du quartier de Gorbitz à Dresde, les personnes en charge de la sécurité routière ont pu s'exprimer et demander des fonds pour améliorer la sécurité routière : «Travailler avec les responsables des projets financés par la Soziale Stadt nous intéresse pour 
Figure 3: Organisation des services administratifs en Allemagne et en France à travers les exemples de Dresde (Saxe) et de Lille (France)

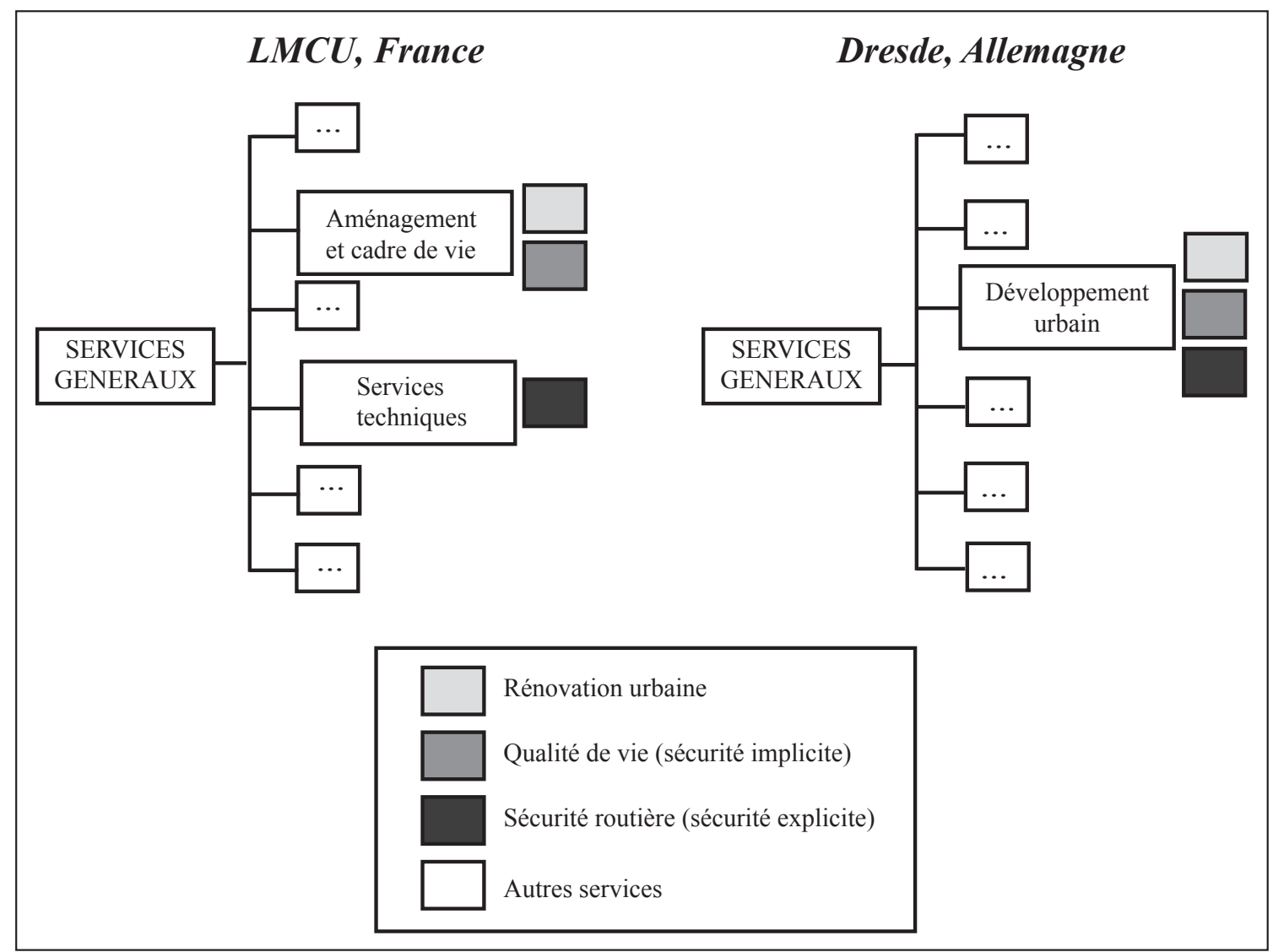

(C) Sylvanie Godillon, 2010

Figure 4 : Conduite des projets de rénovation urbaine à Lille et à Dresde

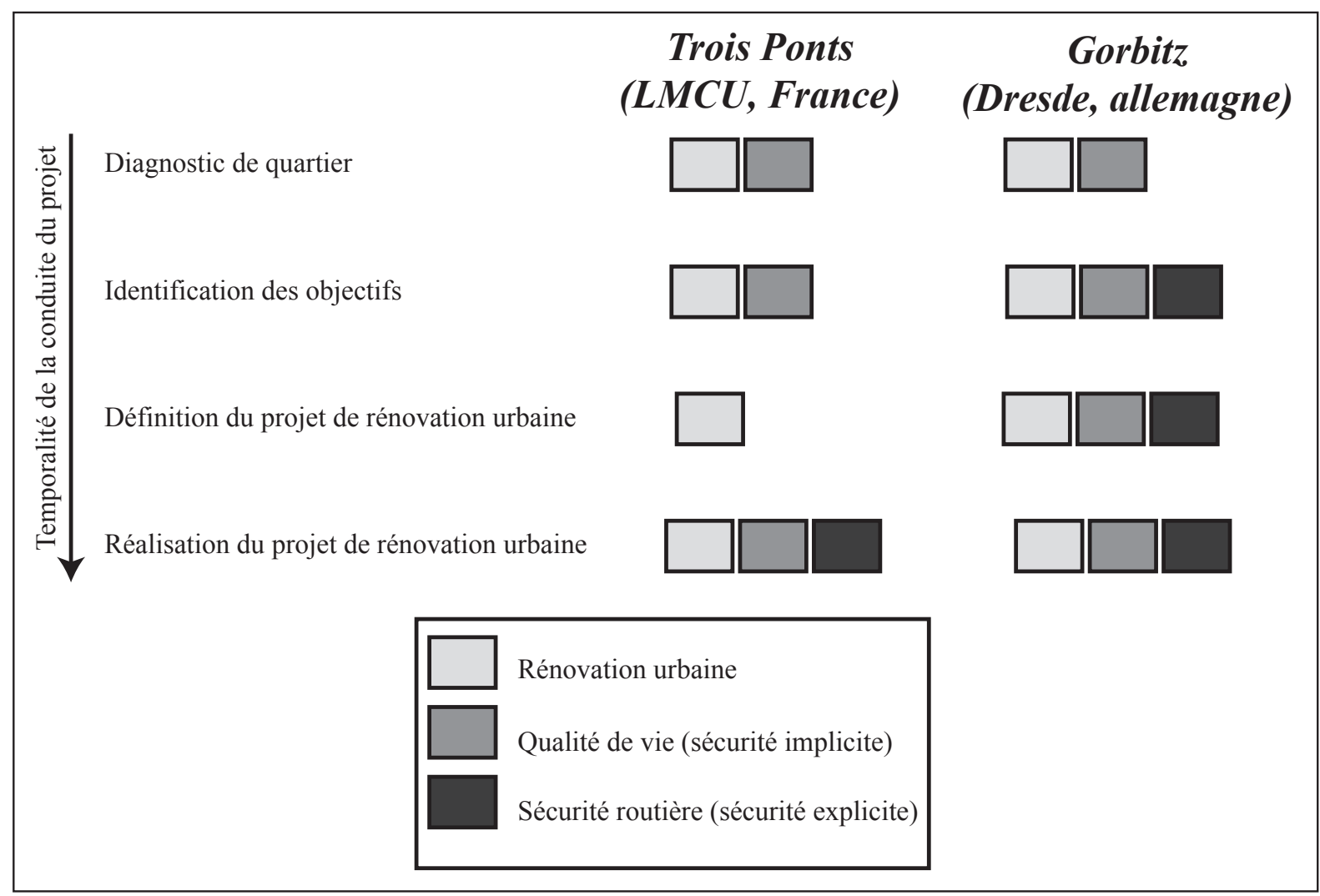

(C) Sylvanie Godillon, 2010 
les financements possibles prévus par ce programme. Mais travailler avec nous les intéresse aussi pour des connaissances sur les dessertes en transport en commun ou les problèmes d'insécurité liés au surdimensionnement des voiries».

Dans les deux cas, les réaménagements des voiries ne s'accompagnent pas d'analyse de l'insécurité routière. En Allemagne, la conduite de projet permet la présence des services s'occupant de la mobilité et des services techniques lors de la définition du projet urbain. En France, la multiplicité des acteurs s'occupant de ces questions rend difficile la confrontation directe des points de vue. La coordination des différents services est assurée par une entité (dans le cas du projet de Roubaix, il s'agit de la Société d'Economie Mixte SEM Ville Renouvelée). Mais les techniciens compétents en matière de sécurité routière n'interviennent qu'après la définition du projet urbain.

\section{CONCLUSION}

En France et en Allemagne, les tendances des deux politiques sectorielles sont semblables : les programmes de rénovation urbaine ont pour objectif d'améliorer les conditions de vie dans les quartiers défavorisés et les politiques de sécurité routière sont orientées vers la responsabilité individuelle. Dans les deux pays, les programmes de rénovation urbaine prônent la transversalité entre les différentes politiques sectorielles comme conditions de réussite. C'est pourquoi l'article s'est centré sur l'analyse de la coordination entre les enjeux de sécurité routière et les projets de rénovation urbaine. La différence majeure entre les deux pays est la plus grande autonomie des acteurs locaux en Allemagne qu'en France.
Dans les études de cas, les projets urbains ne s'accompagnent pas d'une analyse précise de l'accidentologie lors du réaménagement urbain des quartiers. Les différences sont surtout sensibles dans les objectifs du projet urbain et dans la conduite de projet. En Allemagne, les intérêts convergent entre les objectifs de sécurité routière et un programme de rénovation urbaine mettant le trafic en dehors du quartier, alors qu'en France, les intérêts divergent en raison d'une forte volonté de rendre le quartier traversant. Les spécialistes de la sécurité routière sont présents lors des définitions du projet urbain en Allemagne alors qu'ils interviennent dans la mise en ouvre du projet en France. De plus, en France, la multiplicité des acteurs s'occupant de ces questions rend difficile la confrontation directe des différents acteurs d'un même territoire.

La rénovation urbaine est donc une occasion d'intégrer des enjeux de sécurité des déplacements lors du réaménagement des quartiers afin de réduire les inégalités socio-spatiales face au risque d'accident. Si cette occasion est exprimée par les acteurs locaux, elle reste difficile à saisir en France en raison d'une tardive implication des spécialistes de la sécurité routière dans les conduites de projet de rénovation urbaine par rapport à l'Allemagne.

La politique de la ville invente de nouveaux modes d'actions publics (EPSTEIN, 2005). Pour intégrer les enjeux de sécurité routière dans les projets urbains en direction des quartiers défavorisés, plusieurs voies sont possibles : sensibiliser les gestionnaires de projets à ces questions (BERTRAND et al., 2003), intégrer au plus tôt les différentes politiques sectorielles (le cas allemand) ou imposer un diagnostic de sécurité de l'ensemble du quartier lors de projets sur les espaces publics de déplacements. 


\section{Bibliographie}

AGUERO-VALVERDE, J., JOVANIS, P., 2006, « Spatial analysis of fatal and injury crashes in Pennsylvania ", Accident Analysis and Prevention, 38, pp. 618-625

AHRENS, G.-A., 2008, "Integrierte VEP - Anspruch und Wirklichkeit ", Sonderheft der Zeitschrift Internationales Verkehrswesen, 100 Jahren DVWG, pp. $147-153$

BECKER, H., FRANKE T., LOHR R.P., SCHULERI-HARTJE U.-C., 2003, Le programme "Ville sociale": $d u$ renouvellement urbain traditionnel au développement urbain intégratif, traduit de : Stratégies pour la Ville Sociale, Expériences et perspectives - mise en œuvre du programme "Ville sociale", compte rendu final scientifique, Berlin : DIFU, 63 p.

BERTRAND, D., FOUlTIER, C., ROBERT, C., 2003, L'inscription d'un volet "sécurité" routière dans les actions en lien avec la Politique de la Ville, Paris : FORS. Recherche Sociale, 83 pp.

BLANC, M., 2006, "Politique de la ville et Soziale Stadt, une comparaison franco-allemande ", Pensées Plurielles, 12, 2, pp. 45-51

COMMISSION EUROPEENNE, 2001, Livre Blanc : la politique européenne des transports à l'horizon 2010 : l'heure des choix, Luxembourg: Office des publications officielles des Communautés européennes, $135 \mathrm{pp}$.

DESIRE, J.C., MONTEL, M.C., 2000, "Quelle place pour la sécurité routière dans les plans de déplacements urbains? Entre intégration et diffusion, le cas du plan de déplacements urbains de Lille métropole ", Recherche Transport Sécurité, 69, pp. 53-66

DIFU (Deutschen Institut für Urbanistik), 2003, Strategien für die Soziale Stadt Erfahrungen und Perspektiven - Umsetzung des Bund-Länder-Programms "Stadtteile mit besonderem Entwicklungsbedarf - die soziale Stadt ", Berlin : DIFU, 332 p.

EPSTEIN, R., 2005, «Acte II, scène première : la fin de la politique de la ville? Au crible de la loi Borloo", Informations sociales, 121, 1, pp. 88-97

FLEURY, D., 1998, Sécurité et urbanisme. La prise en compte de la sécurité routière dans l'aménagement, Paris : Presse de l'ENPC, 299 p.
GALLEZ, C., GUERRINHA, C., KAUFMANN, V., MAKSIM, H.-N., THEBERT, M., 2008, Mythe et réalités de la cohérence urbanisme-transport, Paris : Rapport de convention 6t-CNRS, $143 \mathrm{p}$.

HASSENTEUFEL, P., 2005, "De la comparaison internationale à la comparaison transnationale. Les déplacements de la construction d'objets comparatifs en matière de politiques publiques ", Revue française de science politique, 55, 1, pp. 113-132

HEWSON, P., 2004, " Deprived children or deprived neighbourhoods? A public health approach to the investigation of links between deprivation and injury risk with reference to child road safety in Devon County ", United Kingdom, BMC Public Health, 4, 15, pp. $1-10$

JACQUIER, C., 2003, Un dispositif de veille internationale sur les approches intégrées de développement urbain en Europe. Rapport de synthèse des politiques intégrées de développement urbain Allemagne, Paris : Délégation Interministérielle à la Ville, $61 \mathrm{p}$.

JOLY, M.F., FOGGIN, P., PLESS, I., 1991, «Les déterminants socio-écologiques du risque d'accident du jeune piéton ", Epidémiologie Santé Publique, 39, 4, pp. 345-351

KOSSMAN, I., 2002, " Le contrôle des vitesses par les autorités locales en Allemagne ", Les Annales des Ponts et Chaussées, 101, pp. 31-37

LIMA, L., STEFFEN, M., 2004, "Comparaisons internationales en politiques publiques : stratégies de recherche, méthodes et interprétation ", Revue Internationale de Politiques Comparées, 11, 3, pp. 339-348

MILLOT, M., 2003, Développement urbain et insécurité routière : l'influence complexe des formes urbaines, Thèse de doctorat, Paris : Ecole Nationale des Ponts et Chaussées, $418 \mathrm{p}$.

PRESTON, B., 1972, "Statistical analysis of child pedestrian accidents in Manchester and Salford ", Accident Analysis and Prevention, 4, pp. 323-332

VAN ELSLANDE P, ALBERTON L, NACHTERGAELE C, BLANCHER G., 1997, Scénarios-types de production de "l'erreur humaine" dans l'accident de la route, Rapport de recherche INRETS n 218 , Arcueil : INRETS, $180 \mathrm{p}$. 